\title{
REVISITING THE SAVANNA HYPOTHESIS: EFFECTS OF HABITAT DISTURBANCE ON HOMINID EVOLUTION
}

\author{
POTTS, Richard, Dept. of Anthropology, National Museum of Natural \\ History, Smithsonian Institution, Washington, DC 20560, U.S.A.
}

Major adaptive change is generally considered to arise by long-term directional selection, consistent in its effect over many generations. Habitat-specific hypotheses of human evolution accord well with this idea. Favored by many paleoanthropologists and evolutionary biologists since Darwin, the savanna hypothesis states that early hominid traits were selected in response to increasingly open and dry habitats. Pliocene and Pleistocene refinements in terrestrial bipedality, tool manufacture, environmental manipulation, cognitive function, and culture have all been assigned to advantages accrued during the shift from forested to open habitats. Recent versions of this hypothesis focus on episodic drying and cooling during the Pliocene, and state that key events in hominid evolution (both turnover and adaptive shifts) occurred in response to these events.

However, the climatic records thought to document this shift toward open habitats actually show an increase in the range of climatic variability over the span of hominid evolution. Hominid lineages, in fact, experienced the Cenozoic's most dramatic fluctuations in climate, vegetation, and resource regimes. Besides climatic oscillation, volcanic and tectonic forces were also sources of long-term uncertainty in early hominid habitats.

According to a new hypothesis, major developments in hominid behavior evolved not due to long-term directional selection in relation to an environmental trend, but by a process of accommodating to habitat variability. The process of variability selection is discussed, whereby key hominid traits were favored in response to long-term disparities in the habitat-specific effects of natural selection. Early australopithecine bipedality, Pliocene origins of the archeological record, Pleistocene increase in brain size, and evidence of hominid behavioral flexibility and diversity all corresponded with long-term extremes in habitat variability.

The applicability of this hypothesis to other organisms is tested using data on Pleistocene large mammals of the southern Kenya rift. Species comparisons indicate that lineages possessing versatile dietary and social strategies outsurvived related large-bodied, dietary specialists during a period of intense environmental fluctuation. Thus other large mammals seem to have paralleled the evolutionary response of hominids to longterm environmental extremes. The savanna or any other habitat-specific hypothesis of hominid evolution should be revised to include the effects of environmental variability. 